\title{
An $H^{\infty}$ Approach to Mitigate the Effects of Array Uncertainties on the MuSIC Algorithm
}

\author{
T. Ratnarajah, Member, IEEE, and A. Manikas, Member, IEEE
}

\begin{abstract}
In this letter, a novel robust technique is proposed to mitigate the degrading effects of finite sampling and imprecise modeling on the performance of the multiple signal classification (MuSIC) algorithm. Initially, a new array-signal state space model is developed, which is valid for any array geometry. Based on this model, formulated in an $H^{\infty}$ framework, a new robust direction-finding approach has been proposed. This theoretical framework is supported by simulation examples that demonstrate the robustness of the new technique to the presence of array uncertainties and signal correlation.
\end{abstract}

Index Terms-Array uncertainties, direction finding, $H^{\infty}$.

\section{INTRODUCTION}

$\mathbf{I}$ T IS WELL known that the performance of the multiple signal classification (MuSIC) algorithm [1] severely degrades due to finite sampling effects and uncertainties such as imprecise modeling of the antenna array and spatial noise statistics ([2]-[7] and references therein). The array uncertainties may be due to sensor position errors, gain errors, phase errors, mutual coupling between sensors, etc. This problem has been conventionally addressed using calibration techniques with limited success.

The goal of this paper is to design a robust direction finding (DF) algorithm, which performs well even in the presence of array uncertainties. One method of addressing the problem is the $H^{\infty}$ approach, which was introduced in robust control theory on the hypothesis that the resulting minimax estimation techniques would be more robust and less sensitive to model uncertainties and parameter variations than conventional techniques. These methods safeguard against the worst-case disturbances and therefore make no assumptions on the (statistical) nature of the signals. This replaces the method of modeling the disturbance signal as a random process with a given spectral density, a method that has led to the well-known linear quadratic regulator and Kalman filter, etc. The performance of these classical estimation methods (such as Kalman filter) heavily depends upon the validity of these model assumptions and hence limits their application.

To apply $H^{\infty}$ estimation techniques to array signal processing, a new state space model for the received signal of a general array of sensors is developed which, in contrast to

Manuscript received September 6, 1996. The associate editor coordinating the review of this manuscript and approving it for publication was Prof. $\mathrm{K}$. Buckley.

The authors are with the Department of Electrical and Electronic Engineering, Imperial College of Science, Technology, and Medicine, University of London, London SW7 2BT, U.K. (e-mail: a.manikas@ic.ac.uk).

Publisher Item Identifier S 1070-9908(98)05137-2. existing models, is capable of handling the simultaneous presence of different types of uncertainties. Then, in Section III, based on this state-space model, a new robust DF technique is proposed. In Section IV, a number of simulation results are illustrated that demonstrate its robustness to calibration errors and signal correlation. Finally, in Section V, the paper is concluded.

\section{Modeling the ARray Signals}

Consider an uncalibrated (or partially calibrated) array of $N$ sensors operating in the presence of $M$ narrowband signals. The received signal vector $\underline{x}(t)$ can be modeled as

$$
\underline{x}(t)=(\mathbf{I}+\tilde{\mathbf{C}})((\mathbf{G}+\tilde{\mathbf{G}}) \odot(\mathbf{A}+\tilde{\mathbf{A}})) \underline{m}(t)+\underline{n}(t)
$$

where

$\odot$ denotes Hadamard product

$m(t)$ message vector-signal

$\underline{n}(t)$ additive white Gaussian noise

$\widetilde{\mathbf{C}}$ mutual coupling uncertainty matrix

$\tilde{\mathbf{G}}$ array gain and phase error matrix

$\tilde{\mathbf{A}}$ sensor position error matrix.

In (1), $\mathbf{G}$ and $\mathbf{A}$ are $N \times M$ complex matrices with their $i$ th column defined as $\gamma\left(\theta_{i}, \phi_{i}\right) \odot e^{j \underline{\psi}\left(\theta_{i}, \phi_{i}\right)}$ and $e^{-j \mathbf{r}^{T} \underline{k}_{i}}$, respectively, where

$$
\underline{k}_{i}=\frac{2 \pi}{\lambda}\left[\cos \left(\theta_{i}\right) \cos \left(\phi_{i}\right), \sin \left(\theta_{i}\right) \cos \left(\phi_{i}\right), \sin \left(\phi_{i}\right)\right]^{T},
$$

$\mathrm{r}$ is the array location matrix, and the vectors $\underline{\gamma}\left(\theta_{i}, \phi_{i}\right)$ and $\psi\left(\theta_{i}, \phi_{i}\right)$ denote the gain and phase response of the array elements for a signal incident from azimuth $\theta_{i}$ and elevation $\phi_{i}$. In this investigation, (1) will be used as the starting point to arrive at an alternative modeling and then to propose a new approach for improving the performance of signal-subspace type DF systems operating in the presence of array uncertainties. The assumption is simply that both the array uncertainties and the noise signals can be considered as bounded energy signals. This implies that the effects due to the presence of array uncertainties can be reduced by using a minimax optimal estimation algorithm, or more specifically, an $H^{\infty}$ optimal approach.

\section{Proposed State Space Model and RoBUSt Direction Finding AlgORITHM}

The proposed state space model is based on the array signal vector $\underline{x}(t)$ given by (1). By reorganizing this equation, the direction gain and phase errors (which need not be identical 


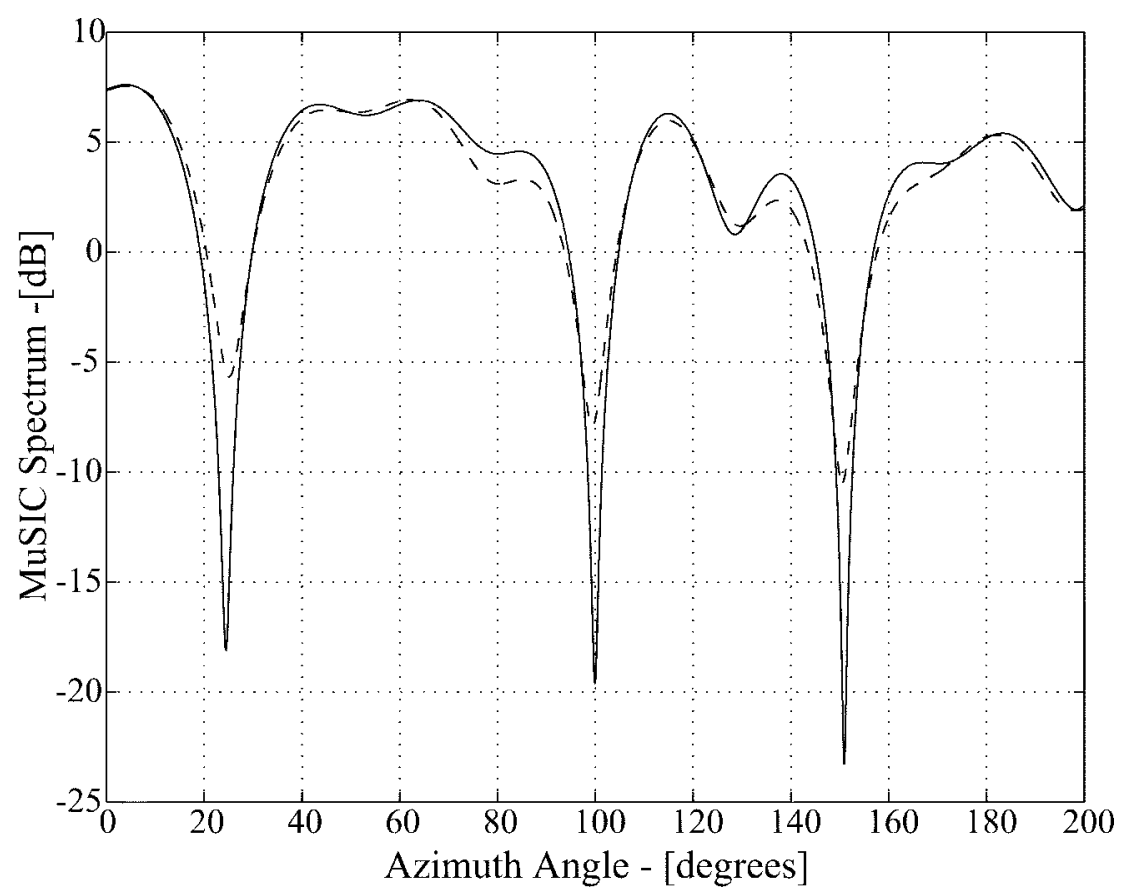

Fig. 1. MuSIC spectrum before (dashed line) and after (solid line) the application of the proposed algorithm.

from sensor to sensor) may be grouped together and be represented, for the $j$ th sensor, by a scalar $v_{j}$. Furthermore the array location errors, mutual coupling and noise effects may be grouped together in an $M \times 1$ vector $\underline{u}_{j}$. In state-space terminology, the scalar $v_{j}$ is the measurement noise while the vector $\underline{u}_{j}$ is the process noise associated with the $j$ th sensor. In this case the elements $x_{j}, j=1, \cdots, N$, of the received signal $\underline{x}(t)$ at a particular time $t$ obey the following state space model:

$$
\begin{aligned}
\underline{s}_{j+1} & =\operatorname{diag}\left[\underline{a}_{j+1} \odot \underline{a}_{j}^{*}\right] \underline{s}_{j}+\mathbf{B}_{j} \underline{u}_{j}, \quad j \in[0, N-1], \\
x_{j} & =\underline{g}_{j}^{T} \underline{s}_{j}+v_{j}, \quad \text { measurement, } j \in[1, N] .
\end{aligned}
$$

$$
\text { where }\left\{\begin{array}{l}
\underline{s}_{j} \in \mathcal{C}^{M \times 1} \text { denotes the state of the } j \text { th-sensor } \\
\underline{g}_{j}=[j \text { th row of } \mathbf{G}]^{T} \\
\underline{a}_{j}= \begin{cases}{[j \text { th row of } \mathbf{A}]^{T}} & j \neq 0 \\
{[1, \cdots, 1]^{T}} & j=0 .\end{cases}
\end{array}\right.
$$

In (2) the matrix $\left(\operatorname{diag}\left[\underline{a}_{j+1} \odot \underline{a}_{j}^{*}\right]\right) \in \mathcal{C}^{M \times M}$ is known as the state transition matrix, while $\mathbf{B}_{j}=\rho \cdot \mathbf{I} \in \mathcal{R}^{M \times M}$, where $\rho$ is a positive constant that imposes a bound on the process noise $\underline{u}_{j}$.

It is important to point out that we make no assumption on the nature of the disturbances (e.g., normally distributed, uncorrelated, etc.) but the proposed new general model assumes that the source directions are loosely known (e.g., supplied by an uncalibrated DF system).

By using the above state space model, the objective is to estimate a new signal $\underline{\underline{z}}(t)$ by "filtering out" the uncertainties from the array signal $\underline{x}(t)$. Let $\mathcal{F}$ be the functional that represents this estimation process, i.e., let

$$
\hat{z}_{i}=\mathcal{F}\left(x_{1}, x_{2}, \cdots x_{i}\right)
$$

which indicates that the $i$ th element $\hat{z}_{i}$ of the new estimated signal $\underline{\hat{z}}(t)$ is a function of the received signals at time $t$ from sensor-1 (i.e., $x_{1}$ ) up to and including the sensor- $i$ (i.e., $x_{i}$ ). Now, let us form the error

$$
e_{i}=\hat{z}_{i}-\underline{g}_{i}^{T} \underline{s}_{i}
$$

and then define the transfer operator $T_{i}(\mathcal{F})$ that maps the unknown disturbances

$$
\left\{\Pi_{0}^{-1 / 2}\left(\underline{s}_{0}-\underline{\hat{s}}_{0}\right), \underline{u}_{1}, \underline{u}_{1}, \cdots, \underline{u}_{i}, v_{1}, v_{2}, \cdots, v_{i}\right\}
$$

to the filtered error

$$
\left\{e_{1}, e_{2}, \cdots, e_{i}\right\}
$$

where $\underline{\underline{s}}_{0}$ denotes an initial guess for $\underline{s}_{0}$, and $\Pi_{0}$ is a given positive definite matrix reflecting a priori knowledge of how close $\underline{s}_{0}$ is to $\underline{\hat{s}}_{0}$. Then the $H^{\infty}$ problem can be restated as "choose the functional $\mathcal{F}(\cdot)$ so as to minimize the $H^{\infty}$ norm of the transfer operator $T_{i}(\mathcal{F})$ " and can be formally presented as follows.

Problem: Given a scalar $\epsilon>0$, find an $H^{\infty}$ suboptimal estimator $\hat{z}_{i}=\mathcal{F}\left(x_{1}, x_{2}, \cdots x_{i}\right)$ which satisfies $\left\|T_{i}(\mathcal{F})\right\|_{\infty}<$ $\epsilon$, or, equivalently, satisfies

$$
\begin{aligned}
& \sup _{\underline{s}_{0}, \underline{u}, v \in h_{2}} \\
& \frac{\sum_{j=1}^{i} e_{j}^{*} e_{j}}{\left(\underline{s}_{0}-\underline{s}_{0}\right)^{H} \Pi_{0}^{-1}\left(\underline{s}_{0}-\underline{s}_{0}\right)+\sum_{j=1}^{i} \underline{u}_{j}^{H} \underline{u}_{j}+\sum_{j=1}^{i} v_{j}^{*} v_{j}}<\epsilon^{2}
\end{aligned}
$$

Solutions: The solution to the above problem can be found by using [8, Th. 1], which indicates that the signal $\hat{z}_{j}$ 
can be estimated as follows:

$$
\hat{z}_{j}=\underline{g}_{j}^{T} \hat{\underline{s}}_{j}
$$

where $\hat{\underline{s}}_{j}$ is recursively computed using the following expression:

$$
\begin{aligned}
\underline{\hat{s}}_{j+1}= & \operatorname{diag}\left[\underline{a}_{j+1} \odot \underline{a}_{j}^{*}\right] \underline{\hat{s}}_{j} \\
& +\underline{K}_{j+1}\left(x_{j+1}-\underline{g}_{j+1}^{T} \operatorname{diag}\left[\underline{a}_{j+1} \odot \underline{a}_{j}^{*}\right] \underline{\hat{s}}_{j}\right)
\end{aligned}
$$

$$
\begin{aligned}
& \text { with }\left\{\begin{aligned}
\underline{\hat{s}}_{0}= & \text { initial guess }=\text { TLS solution of } \underline{x}(t) \\
= & (\mathbf{G} \odot \mathbf{A}) \underline{s}(t) \\
\underline{K}_{j+1}= & \mathbf{P}_{j+1} \underline{g}_{j+1}^{*}\left(1+\underline{g}_{j+1}^{T} \mathbf{P}_{j+1} \underline{g}_{j+1}^{*}\right)^{-1} \\
\mathbf{P}_{j+1}= & \operatorname{diag}\left[\underline{a}_{j+1} \odot \underline{a}_{j}^{*}\right] \mathbf{P}_{j} \operatorname{diag}\left[\underline{a}_{j+1} \odot \underline{a}_{j}^{*}\right]^{H} \\
& +\mathbf{B}_{j} \mathbf{B}_{j}^{H}-\operatorname{diag}\left[\underline{a}_{j+1} \odot \underline{a}_{j}^{*}\right] \mathbf{P}_{j}\left[\underline{g}_{j}^{*} \quad \underline{g}_{j}^{*}\right] \\
& \left.\times \mathbf{R}_{e, j}^{-1}\left[\underline{g}_{j}^{T}\right] \underline{g}_{j}^{T}\right] \mathbf{P}_{j} \operatorname{diag}\left[\underline{a}_{j+1} \odot \underline{a}_{j}^{*}\right]^{H} \\
&
\end{aligned}\right. \\
& =\text { Riccati recursion equation } \\
& \mathbf{R}_{e, j}=\left[\begin{array}{cc}
1 & 0 \\
0 & -\epsilon^{2}
\end{array}\right]+\left[\begin{array}{l}
\underline{g}_{j}^{T} \\
g_{j}^{T}
\end{array}\right] \mathbf{P}_{j}\left[\begin{array}{ll}
g_{j}^{*} & \underline{g}_{j}^{*}
\end{array}\right] \\
& \mathbf{P}_{0}=\mathbf{\Pi}_{0}, \mathbf{B}_{0}=\rho \mathbf{I}, \underline{g}_{0}=[1, \cdots, 1]^{T} .
\end{aligned}
$$

Note that for a given $\epsilon>0$ and if the transition matrices are nonsingular (in our case the matrices $\operatorname{diag}\left[\underline{a}_{j+1} \odot \underline{a}_{j}^{*}\right]$ are always nonsingular), then the above solution exists if and only if

$$
\mathrm{P}_{j}^{-1}+\left(1-\epsilon^{-2}\right) \underline{g}_{j}^{*} \underline{g}_{j}^{T}>0, \quad j=0, \cdots, i .
$$

We shall assume, without loss of generality, that $\Pi_{0}$ has the special form $\Pi_{0}=\mu \mathbf{I}$, where $\mu$ is a positive constant.

Using this above $H^{\infty}$ a posteriori approach, a new algorithm is derived based on processing the array output data matrix of $L$ snapshots $\mathbf{X}=\left[\underline{x}\left(t_{1}\right), \cdots, \underline{x}\left(t_{L}\right)\right]$ to get the data matrix $\mathbf{Z}=\left[\underline{z}\left(t_{1}\right), \cdots, \underline{z}\left(t_{L}\right)\right]$. The proposed algorithm can be presented in a step format as follows.

1) Form the data covariance matrix, $\mathbf{R}_{x x}=\frac{1}{L} \mathbf{X X}^{H}$.

2) Based on $\mathbf{R}_{x x}$ and the nominal array manifold parameters, estimate the directions of arrival (DOA's) $\left\{\hat{\theta}_{i}, \hat{\phi}_{i}, i=1, \cdots, M\right\}$, using the MuSIC algorithm.

3) From the estimated DOA's, formulate the proposed model and estimate the filtered signal $\underline{z}(t)=\left[z_{1}, \cdots, z_{N}\right]^{T}$ using (5) and (6). Similarly, estimate the matrix $\mathbf{Z}$ and form the filtered data covariance matrix $\mathbf{R}_{z z}$.

4) Reestimate the DOA's $\left\{\theta_{i}, \phi_{i}, i=1, \cdots, M\right\}$, based on $\mathbf{R}_{z z}$ and repeat 3) a few times (in the simulation examples below we repeat five times) using the previously filtered data.

Remark: In order to construct the $H^{\infty}$ a posteriori filter, we need to know the source directions, which are unavailable. However, we show that the source directions need to be known only approximately. This suggests the possibility of
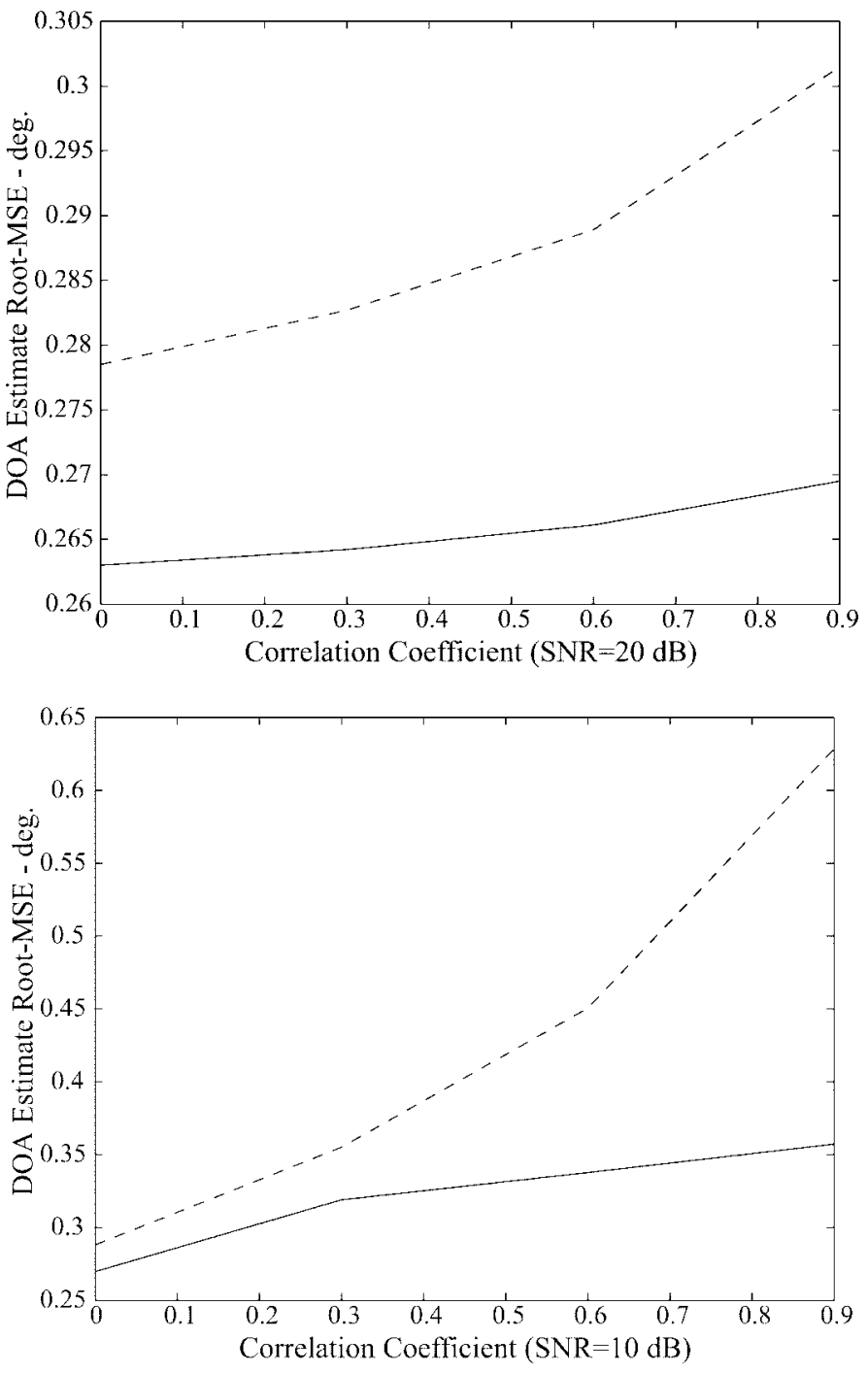

Fig. 2. Two correlated sources-RMSE versus correlation coefficient for the source at $70^{\circ}$, where $\Delta \theta=6^{\circ}, \mathrm{SNR}=20$, and $10 \mathrm{~dB}$ and array manifold error covariance is given by (8). Dashed line: MuSIC algorithm. Solid line: proposed algorithm.

using a repetitive processing scheme in which initial direction estimates are used to design the $H^{\infty}$ a posteriori filter and subsequently obtain improved direction estimates.

\section{Simulation Examples}

Consider the planar circular array of six isotropic antennas, with antenna-locations described by the following matrix (in meters):

$$
\begin{aligned}
& \mathbf{r}= \\
& {\left[\begin{array}{cccccc}
15.22 & 24.79 & 9.57 & -9.57 & -23.10 & -23.10 \\
19.84 & -3.27 & -23.10 & -23.10 & -9.57 & 9.57 \\
0 & 0 & 0 & 0 & 0 & 0
\end{array}\right] .}
\end{aligned}
$$

We assumed that the array operates in the presence of mutual coupling effects (uncertainties) with each sensor significantly coupled with its nearest neighbors, while the coupling with other sensors can be ignored. Furthermore, consider that 
there are also gain, phase, and location errors, which can be described as follows:

$$
\begin{aligned}
\tilde{\gamma}= & {[0,0.1530,0.2211,0.1207,0.0653,0.0165]^{T} } \\
\tilde{\psi}= & {\left[0^{\circ}, 1.7762^{\circ}, 1.9824^{\circ}, 3.0653^{\circ}, 1.7017^{\circ}, 9.8033^{\circ}\right]^{T} } \\
\tilde{\mathbf{r}}= & {\left[\begin{array}{cccccc}
0 & 0.0668 & 0.6872 & 0.9305 & 0.5266 & 0.6543 \\
0 & 0.4179 & 0.5894 & 0.8457 & 0.0918 & 0.4159 \\
0 & 0 & 0 & 0 & 0 & 0
\end{array}\right] } \\
& \text { in meters. }
\end{aligned}
$$

Fig. 1 illustrates the results (solid line) of the proposed algorithm for the above configuration with the array operating in the presence of three uncorrelated equipower sources at $\left(25^{\circ}, 0^{\circ}\right),\left(100^{\circ}, 0^{\circ}\right)$, and $\left(150^{\circ}, 0^{\circ}\right)$. The background noise power is $20 \mathrm{~dB}$ below the source power and the received signal is formed from 100 snapshots. The nominal gain and phase of the antennas were assumed to be one and zero, respectively. It is clear that the array uncertainty effects are reduced after the application of the proposed algorithm and approximately $15 \mathrm{~dB}$ improvement is achieved.

Finally, two computer simulated experiments were carried out using a ten element (nominally) uniform linear array. The signal environment involves two equipower sources located at $66^{\circ}$ and $70^{\circ}$, and it was assumed that the number of emitters $M$ had been correctly determined in all of the examples. In every experiment, 200 runs are performed, and the root mean square error (RMSE) for each direction is estimated. A perturbation (bound) modeled as independent, circular, complex random variables with variance

$$
\begin{aligned}
& \mathcal{E}\left\{\underline{\tilde{a}}\left(\theta_{i}\right) \cdot \underline{\tilde{a}}^{H}\left(\theta_{j}\right)\right\}=\operatorname{diag}\{0.1,0.1,0.01, \cdots, 0.01,0.1,0.1\} \\
& \mathcal{E}\left\{\underline{\tilde{a}}\left(\theta_{i}\right) \cdot \underline{\tilde{a}}^{T}\left(\theta_{j}\right)\right\}=\mathbf{0} \quad \forall i, j .
\end{aligned}
$$

was made to the array response. Though the sensor errors themselves are angle dependent, their statistics under this model are independent of $\theta$.

Fig. 2 shows the RMSE versus signal correlations for 20 and $10 \mathrm{~dB}$ SNR. The solid line represents the proposed algorithm and the dashed line represents the MuSIC algorithm. From the figures, it is clear that the proposed algorithm reduces the
RMSE and, hence, is robust to calibration uncertainties. In the worst-case scenario, where signal-to-noise ratio (SNR) is equal to $10 \mathrm{~dB}$ and the correlation coefficient is equal to 0.9 , MuSIC was unable to resolve the two sources 26 times. These trials were eliminated from the RMSE calculation. It is clear from the results that the proposed algorithm not only enhances the performance (sharpness of the MuSIC spectrum) but also reduces the RMSE (better accuracy).

\section{CONCLUSION}

In this letter, a new array-signal state space model has been developed for a general array geometry. Using this model, a new algorithm has been proposed, based on $H^{\infty}$ estimation theory, which mitigates the simultaneous degrading effects of finite sampling and imprecise modeling of the antenna array and spatial noise statistics on the performance of the MuSIC algorithm. The performance difference (based on RMSE) between the proposed and MuSIC algorithms was shown to be greatest in situations with low SNR and high signal correlation.

\section{REFERENCES}

[1] R. O. Schmidt, "Multiple emitter location and signal parameter estimation,"IEEE Trans. Antennas Propagat., vol. 34, pp. 276-280, Mar. 1986.

[2] M. Kaveh and J. Barabell, "The statistical performance of the MuSIC and the minimum-norm algorithms in resolving plane waves in noise," IEEE Trans. Acoust., Speech, Signal Processing, vol. ASSP-34, pp. 331-341, Apr. 1986.

[3] J. Pierre and M. Kaveh, "Experimental performance of calibration and direction-finding algorithms," in Proc. IEEE ICASSP'91, pp. 1365-1368.

[4] B. Friedlander and A. J. Weiss, "Self-calibration for high-resolution array processing," in Advances in Spectrum and Array Processing, Vol. II. S. Haykon, Ed. Englewood Cliffs, NJ: Prentice-Hall, 1991.

[5] A. Manikas and N. Fistas, "Modeling and estimation of mutual coupling between array element," in Proc. IEEE ICASSP'94, pp. 77-80.

[6] M. Viberg and A. Swindlehurst, "Analysis of the combined effects of finite samples and model error on array processing performance," IEEE Trans. Signal Processing, vol. 42, pp. 3073-3083, Nov. 1994.

[7] M. Viberg and A. L. Swindlehurst, "A Bayesian approach to autocalibration for parametric array signal processing," IEEE Trans. Signal Processing, vol. 42, Dec. 1994.

[8] B. Hassibi, A. H. Sayed, and T. Kailath, "Linear estimation in Krein spaces-Part I: Theory and Part II: Applications," IEEE Trans. Automat. Contr., vol. 41, pp. 18-49, Jan. 1996. 